\title{
Implanted islets in the anterior chamber of the eye are prone to autoimmune attack in a mouse model of diabetes
}

\author{
Majid Mojibian • Brigitte Harder • Andrew Hurlburt • \\ Jennifer E. Bruin • Ali Asadi • Timothy J. Kieffer
}

Received: 10 April 2013 / Accepted: 4 July 2013 / Published online: 11 August 2013

(C) Springer-Verlag Berlin Heidelberg 2013

\begin{abstract}
Aims/hypothesis Type 1 diabetes is an autoimmune disease resulting from the destruction of insulin-producing beta cells. Along with advances in generating replacement beta cells for treating diabetes, there is also increasing demand for non-invasive tools to evaluate the recurrence of autoimmune attack on transplanted tissue. Here, we examined the anterior chamber of the eye as a potential islet transplant site, and also evaluated whether in vivo imaging of the islets transplanted in the eye could enable real-time visualisation of autoimmune processes underway in the pancreas.

Methods Syngeneic islet equivalents were transplanted into the eye or kidney capsule of streptozotocin-induced diabetic C57BL/6 mice to compare islet dose (25-125 islet equivalents) and function across transplant sites. Autoimmune attack of syngeneic islets was evaluated in the pancreas and eye tissues of NOD and NOD-severe combined immunodeficient (SCID) mice given diabetogenic splenocytes.

Results Islet transplantation in the eye decreased fasting plasma glucose levels and increased weight gain and survival in an islet-dose-dependent manner. Even 50 islets in the eye reduced blood glucose levels, whereas $\geq 200$ islets were required in the kidney for a similar effect. Autoimmune
\end{abstract}

Electronic supplementary material The online version of this article (doi:10.1007/s00125-013-3004-z) contains peer-reviewed but unedited supplementary material, which is available to authorised users.

M. Mojibian · B. Harder · A. Hurlburt · J. E. Bruin · A. Asadi · T. J. Kieffer $(\bowtie)$

Laboratory of Molecular and Cellular Medicine, Department of

Cellular and Physiological Sciences, Life Sciences Institute,

University of British Columbia, 2350 Health Sciences Mall,

Vancouver, BC, Canada V6T 1Z3

e-mail: tim.kieffer@ubc.ca

T. J. Kieffer

Department of Surgery, University of British Columbia,

Vancouver, BC, Canada destruction of pancreatic islets in the eye mirrored that in the pancreas and could be visualised in real time by noninvasive imaging.

Conclusions/interpretation We found that far fewer islets were required to restore normoglycaemia when transplanted into the anterior chamber of the eye vs the kidney capsule. However, our results suggest that islets are not protected against autoimmune attack in the eye, making this a suitable site for visualising autoimmune processes against transplanted tissue.

Keywords Anterior chamber of the eye Autoimmune attack $\cdot$ Diabetes $\cdot$ Islets $\cdot$ Transplantation

$\begin{array}{ll}\text { Abbreviations } \\ \text { HBSS } & \text { Hanks' balanced salt solution } \\ \text { MSFHR } & \text { Michael Smith Foundation for Health Research } \\ \text { SCID } & \text { Severe combined immunodeficient } \\ \text { STZ } & \text { Streptozotocin }\end{array}$

\section{Introduction}

Type 1 diabetes is a severe, chronic autoimmune disease caused by the loss of self tolerance to insulin-producing pancreatic beta cells, resulting in hyperglycaemia associated with debilitating health complications [1]. Transplantation of insulin-producing cells has shown remarkable potential for treating patients with type 1 diabetes [2, 3]. However, islet transplant recipients require chronic immunosuppression to prevent both allo- and autoimmune destruction of engrafted tissue. It is estimated that more than half of the transplanted islet tissue is lost in the first few days after syngeneic transplantation $[2,3]$. The mechanisms underlying this loss are poorly understood, as monitoring the survival of islets 
transplanted into deep tissues such as the liver remains a clinical challenge despite recent advances [4, 5].

In rodents, pancreatic islets are typically transplanted under the kidney capsule, as this site is easily accessible, highly vascularised and retrievable via survival nephrectomy. Islet graft function can be assessed non-invasively by measuring circulating glucose and C-peptide levels. It is important, however, to consider that both engrafted cells and the endogenous pancreas secrete C-peptide. Therefore, a xenotransplant can be used to specifically assess graft-derived C-peptide levels using species-specific assays. Moreover, direct visualisation of transplanted tissue typically requires an invasive surgical procedure to expose the kidney for imaging and is not practical for longitudinal, repeated imaging and tracking of single cells [6].

Recently, various imaging techniques for evaluating the fate of transplanted islets and the progression of autoimmune diabetes have been examined [5, 7-12]. Among these, optical imaging provides an attractive imaging modality because it is a non-invasive and non-ionising technique [13] and can track live cells in vivo by fluorescent or bioluminescent labelling. Fluorescent imaging is hampered by two major limitations: insufficient penetration depth of most fluorescent agents and inadequate resolution resulting from the high absorption and broad autofluorescence of tissues [13]. Even using near-infrared contrast agents or tissue-specific bioluminescence [10-12], these techniques are not able to attain single-cell-level sensitivity [14]. The anterior chamber of the eye has been a popular transplantation site for decades [15], as transplanted tissue is readily vascularised and innervated by the iris [16] and the graft can be visualised noninvasively. As such, specific techniques have been developed for transplantation and imaging of transplanted tissue in the eye [16-19]. Despite a general belief that the anterior chamber is an immune privileged area due to its anatomical structure and composition of immune cells and soluble factors [20-22], intraocular islet transplantation has been shown to be well-suited for studying alloimmune responses against pancreatic beta cells [18].

Recently, in vivo imaging of autoimmune destruction of endogenous pancreatic islets was reported at cellular resolution [23]. Mice expressing a viral glycoprotein in beta cells underwent adoptive transfer of $\mathrm{T}$ cell receptor-restricted $\mathrm{CD} 8^{+}$ lymphocytes towards the viral protein, and cytotoxic T lymphocyte activity was imaged via live microscopy of surgically exposed pancreases. While this approach enables visualisation of immune attack against endogenous pancreatic islets, utility for studying autoimmune attack was not demonstrated. Moreover, this surgical technique is impractical for evaluating the slow process of beta cell autoimmune destruction over prolonged periods. The anterior chamber of the eye offers a window into the body, and we hypothesised that it might be a feasible site for non-invasive imaging of beta cell autoimmunity.
In the current study, we first evaluated the capacity of the eye to support islet function in comparison with the kidney capsule by examining the efficacy of multiple allogeneic islet doses for treating streptozocin (STZ)-induced diabetes in C57BL/6 mice. Second, we investigated the feasibility of visualising autoimmune attack of islets in real time through in vivo imaging of islet transplants in the anterior chamber. These studies aimed to determine whether the autoimmune destruction of engrafted islets in the eye mirrored that of islets within the endogenous pancreas.

\section{Methods}

Generation of diabetes in mice All experiments were approved by the University of British Columbia Animal Care Committee in accordance with the guidelines of the Canadian Council of Animal Care. Male C57BL/6, female NODsevere combined immunodeficient (SCID) and female NOD mice ( $8-10$ weeks old) were obtained from Jackson Laboratory (Bar Harbour, ME, USA). Mice were housed in the Centre for Disease Modeling facility (Vancouver, BC, Canada) in a climate-controlled room with a $12 \mathrm{~h}$ light-dark cycle and provided with water and free access to a standard irradiated diet (Teklad Diet No. 2918; Harlan Laboratories, Madison, WI, USA). Blood glucose and body weight were monitored once or twice weekly following a $4-5 \mathrm{~h}$ morning fast. Blood glucose levels were measured via the saphenous vein using a handheld glucometer (LifeScan, Burnaby, BC, Canada). Diabetes was generated in C57BL/6 mice by a single intraperitoneal injection of STZ $(180 \mathrm{mg} / \mathrm{kg}$ body weight; Sigma-Aldrich, Oakville, ON, USA) 5-10 days before islet transplantation.

Pancreatic islet isolation and preparation Pancreatic islets were isolated from donor mice 1-2 days before transplantation, using techniques described by Lacy and Kostianovsky [24] with an additional filtration step [25]. After overnight culture, islet numbers, purity and viability were assessed by microscopy (live/dead assay; Invitrogen, Burlington, ON, Canada). Islets were hand-selected and washed three times in sterile PBS. Islets of various sizes were picked into groups of islet equivalents ( 1 islet equivalent $=\sim 50 \mu \mathrm{m}$ in diameter) for transplantation.

Islet transplantation To examine the function of islets in the eye, $25(n=5), 50(n=5), 75(n=6)$ or $125(n=4)$ C57BL/6 islet equivalents were transplanted into the anterior chamber of the eye of C57BL/6 recipient mice with STZ-induced diabetes. The efficacy of different subcapsular islet doses was assessed by transplanting $50(n=3), 125(n=3), 200$ $(n=3)$ or $275(n=2) \mathrm{C} 57 \mathrm{BL} / 6$ donor islets under the left kidney capsule of C57BL/6 mice with STZ-induced diabetes. 
Age-matched C57BL/6 mice with or without STZ-induced diabetes received sham surgery (no islet transplant) in the eye $(n=4)$ or kidney capsule $(n=7)$.

NOD-SCID mice $(n=9)$ received 30 hand-picked islet equivalents (isolated from donor NOD-SCID mice) in the eye 3 weeks before adoptive transfer of splenocytes from newly diabetic NOD mice. A second group of NOD mice $(n=7)$ received syngeneic islet transplants in the eye at 8 weeks of age and diabetes was allowed to progress naturally, without adoptive transfer.

For islet transplantation into the anterior chamber of the eye, islets were drawn into a micropipette (MXL3-BPIND-200; Origio MidAtlantic Devices, Mt Laurel, NJ, USA) using a micromanipulator and settled by gravity. The cornea was punctured obliquely using a $27 \mathrm{G}$ needle, and the needle was passed into the anterior chamber without damaging the iris. The site of entry was widened with heat-sterilised fine forceps, the micropipette containing the islets was passed into the anterior chamber and islets were ejected using the micromanipulator. The pipette was removed and Isoptears (Alcon Canada, Mississauga, ON, Canada) supplemented with gentamicin ( $0.3 \% \mathrm{wt} / \mathrm{vol}$.) was applied to the eye to prevent desiccation and infection.

Splenocyte extraction and adoptive transfer Spleens from newly diabetic NOD mice (4-6 months old, $n=5$ ) were harvested into Hanks' balanced salt solution (HBSS) containing $1 \%$ bovine serum albumin, cut into small pieces and gently mashed between two histology slides. Cells were rinsed with HBSS, filtered with a $0.2 \mu \mathrm{m}$ cell strainer (Fisher Scientific, Ottawa, ON, Canada), centrifuged (5 min at 1,500 rpm), washed with HBSS, centrifuged again and resuspended in lysis buffer (ammonium chloride/Tris- $\mathrm{HCl}, \mathrm{pH}$ 7.5). The cell lysate was centrifuged and washed with HBSS to remove erythrocyte debris. A sample of cells was stained with trypan blue (Invitrogen) and counted via haemocytometry. Twenty-million splenocytes in $100 \mu \mathrm{l}$ were injected into the tail vein of restrained, conscious, female NOD-SCID mice (11 week old, $n=11$ ). Nine of eleven adoptive transfer recipient mice received syngeneic islet transplants in the anterior chamber of the eye 4 weeks earlier.

Non-invasive in vivo imaging of the eye To visualise autoimmune destruction of islets, live images of the engrafted eye were captured using a Leica MacroFluo (Z16 APO A) (Leica Microsystems Canada, Concord, Ontario, Canada) at three time points before adoptive transfer (day -28 , day -8 and day 0 ) and five time points after adoptive transfer (days $5,19,21,25$ and 28).

OGTT At 45 days post transplant in the islet dosing study, islet recipients with fasting plasma glucose $<11 \mathrm{mmol} / \mathrm{l}$ underwent an OGTT following a $6 \mathrm{~h}$ morning fast. Mice received an oral bolus of $30 \%$ glucose $(2 \mathrm{~g} / \mathrm{kg})$ and glucose levels were measured at 15, 30, 60, 90 and 120 min following gavage.

Enucleation At 47 days post transplant in the islet dosing study, the engrafted eye was surgically removed from the subset of mice that received an OGTT $(n=4)$ and agematched non-diabetic control mice $(n=3)$.

Tissue harvest and processing In the islet dosing study, engrafted eyes were harvested from all remaining recipient mice (i.e. non-enucleated) at 65 days post transplant. Engrafted kidneys were harvested at 46 days post transplant. In the adoptive transfer study, pancreases and engrafted eyes were harvested from NOD-SCID mice either 2.5 weeks post transfer of splenocytes, before clinical symptoms of diabetes $(n=3)$, or 4 weeks post adoptive transfer $(n=8$ mice in total: $n=3$ newly diabetic, $n=3$ prediabetic and $n=2$ NOD-SCID sham). Pancreas tissue and engrafted eyes were also harvested from transplanted NOD mice without adoptive transfer after 14 weeks of age, but before they developed diabetes $(n=7)$.

Eyes with engrafted islets were placed in Davidson's fixative (two parts $37 \%$ formalin, three parts $100 \%$ ethanol, one part glacial acetic acid and three parts tap water) for $24 \mathrm{~h}$ at $4{ }^{\circ} \mathrm{C}$, then transferred to $70 \%$ ethanol for storage. Engrafted kidneys and pancreases were stored in $4 \%$ paraformaldehyde for $24 \mathrm{~h}$ at $4^{\circ} \mathrm{C}$, then transferred to $70 \%$ ethanol. Tissues were processed into $5 \mu \mathrm{m}$ paraffin sections and mounted by Wax-it Histology Services (Vancouver, BC, Canada).

Histopathological evaluation of insulitis The severity of insulitis was determined in sections of pancreas and engrafted eyes collected 2 weeks post adoptive transfer (before diabetes) and at diabetes onset. For mice that did not become diabetic, insulitis was assessed at 5 weeks post transfer. For the spontaneous diabetic group, the severity of insulitis was determined when mice were 4-6 months old or at onset of diabetes. Insulitis was evaluated using haematoxylin/eosin-stained sections; immunostaining for insulin, glucagon and synaptophysin was performed in neighbouring sections to mark the endocrine cells.

For pancreas sections, 20-30 islets from three sections (separated by $100 \mu \mathrm{m}$ ) were graded and averaged. Pancreas scoring was based on the system described elsewhere [18]: a score of ' 0 ' represented no immune cells surrounding islets; ' 1 ' represented peri-insulitis (mononuclear cells surrounding the islet periphery); ' 2 ' represented moderate insulitis (mononuclear cells infiltrating $<50 \%$ of islet area) and ' 3 ' represented severe insulitis (mononuclear cells infiltrating $>50 \%$ of islet area).

Immune infiltration grading for the eye was based on the same system. Engrafted islets were located by examining 
sections at different depths from the eye and staining for insulin, glucagon and synaptophysin. Infiltration of mononuclear cells was scored as follows using DAPI-stained sections: ' 0 ' represented no mononuclear cells; ' 1 ' represented few mononuclear cells around the periphery of the transplant; ' 2 ' represented large numbers of infiltrating cells in the islet area and ' 3 ' represented infiltration in $>50 \%$ of the transplanted tissue. CD3 staining was performed to confirm that the infiltrating cells were $\mathrm{T}$ lymphocytes.

Immunofluorescent staining Tissue sections were deparaffinised and immunostained as previously described [26] using primary antibodies as follows: guinea pig anti-insulin (1/1,000; Millipore, Billerica, MA), guinea pig anti-insulin $(1 / 1,000$; Sigma-Aldrich, St Louis, MO, USA), mouse antiglucagon (1/1,000; Sigma-Aldrich, St Louis, MO, USA) and rabbit anti-CD3 (1/50; AnaSpec, San Jose, CA, USA). Stained sections were either viewed with a Zeiss Axiovert 200 inverted fluorescence microscope (Carl Zeiss, Toronto, ON, Canada) connected to a Retiga 2000R Fast 1394 camera (QImaging, Burnaby, BC, Canada) or ImageXpress Micro Automated Acquisition system (Molecular Devices, Sunnyvale, CA, USA). Images were analysed using Openlab v5.0.2 (PerkinElmer, Waltham, MA, USA) or MetaXpress software (Molecular Devices).

Data analysis Numerical data analysis was performed using GraphPad Prism v5.0a (GraphPad Software, San Diego, CA, USA). Statistical difference in growth rate was determined by linear regression followed by comparison of regression slopes via one-way ANOVA. Plasma glucose measurements were analysed using two-way ANOVA followed by Bonferroni multiple comparison tests. Plasma glucose changes in response to the OGTT were analysed using two-way ANOVA and comparison of AUC using a Student's $t$ test. The inter-relationship between the insulitis score in the eye and the pancreas was analysed using a Pearson correlation.

\section{Results}

Anterior chamber of the eye as a site for functional islet transplantation We first investigated the ability of different doses of allogeneic islets (25-125 islet equivalents), transplanted into the anterior chamber of the eye, to reverse STZ-induced diabetes in male C57BL/6 mice, using nondiabetic and sham-treated diabetic mice as controls. The cumulative survival of mice from each recipient group was assessed for the time between transplantation and graft removal (days $0-47$ ) and was positively correlated to the number of islets transplanted (Fig. 1a). During the 47 day study, five mice became moribund and were killed; two of
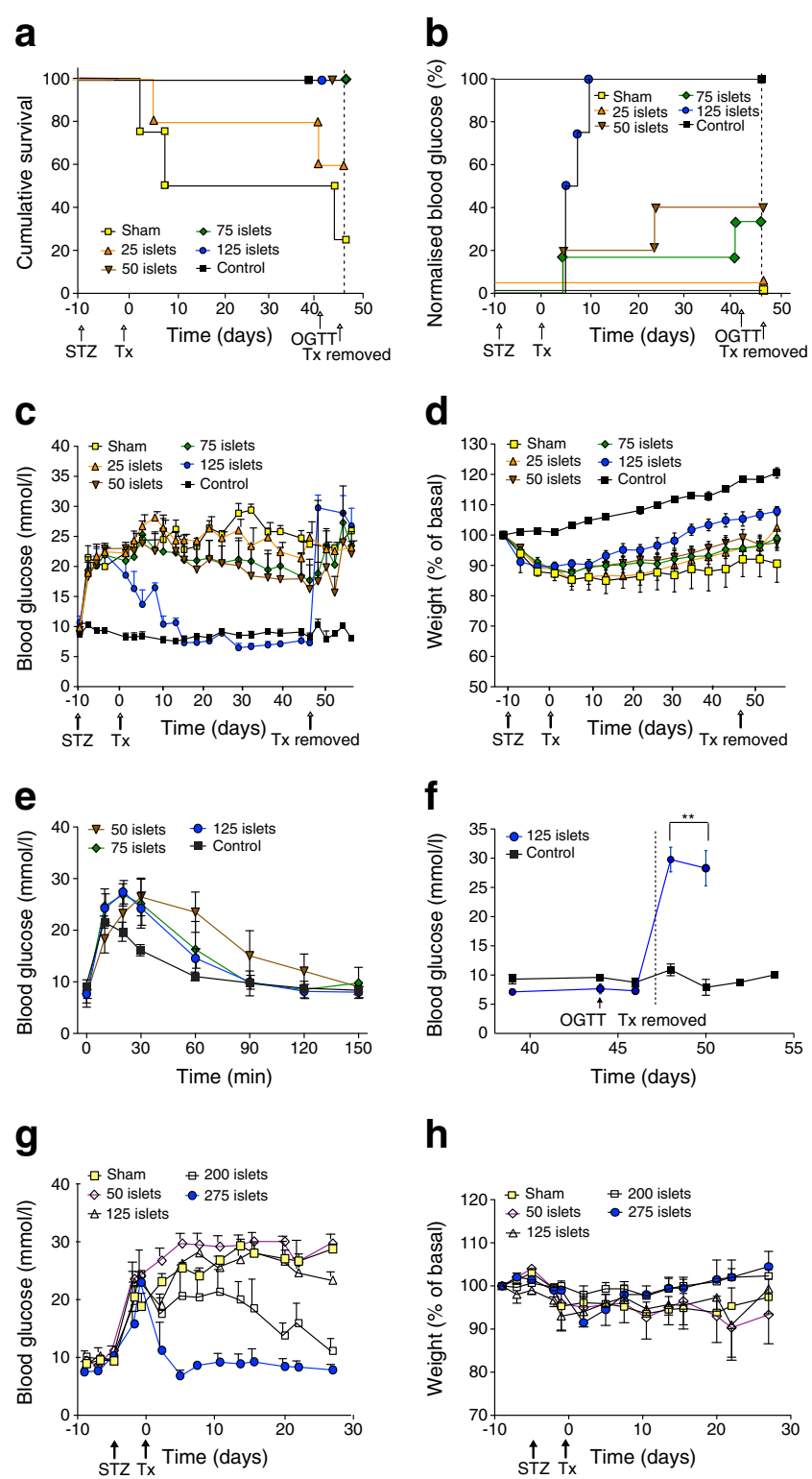

h

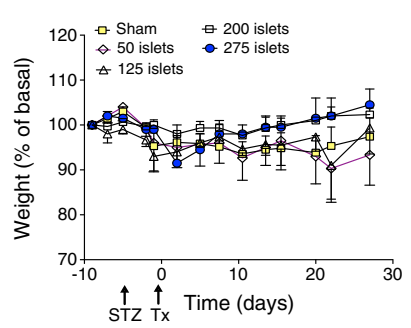

Fig. 1 Reversal of fasting hyperglycaemia in STZ-induced diabetic mice depends on the number of islets transplanted in the anterior chamber of the eye. (a, b) Diabetes survival data and blood glucose normalisation for STZinduced diabetic mice transplanted with $25(n=5), 50(n=5), 75(n=6)$ or $125(n=4)$ islet equivalents in the anterior chamber of the eye are shown vs sham-treated diabetic mice $(n=4)$ and non-diabetic control mice $(n=4)$. Arrows identify the times of STZ treatment (STZ), islet transplantation (Tx) and an OGTT in euglycaemic transplant recipients. (c) Tracking of mean fasted blood glucose concentrations $( \pm$ SEM). (d) Tracking of mean body weights $( \pm \mathrm{SEM})$. (e) Islet transplants in the anterior chamber of the eye improved oral glucose tolerance in STZ-treated mice. (f) Transplanted islets were removed via enucleation from recipient mice $(n=4)$ that became euglycaemic post transplantation. Enucleation of the eye transplanted with islets resulted in fasting hyperglycaemia. ${ }^{* *} p<0.01$, before vs after enucleation of engrafted eye. The dotted line indicates transplant removal. (g, h) Reversal of fasting hyperglycaemia in STZ-induced diabetic mice depends on the number of islets transplanted under the kidney capsule. Tracking of mean blood glucose concentrations ( \pm SEM) (g) and mean body weight $( \pm$ SEM) (h) following a $5 \mathrm{~h}$ morning fast are shown for STZinduced diabetic mice transplanted with $50(n=3), 125(n=3), 200(n=3)$ or $275(n=2)$ islet equivalents in the kidney capsule vs sham-treated diabetic mice $(n=7)$ 
these mice had received transplants of 25 islets ( $60 \%$ survival) and three were diabetic/sham-treated mice ( $25 \%$ survival). All of the mice that received 50,75 or 125 islets, as well as the non-diabetic control mice, survived over the same period $(100 \%$ survival), indicating that transplantation of even 50 islets in the eye improved survival in mice with diabetes (Fig. 1a). Furthermore, the corresponding proportions of mice that experienced reversal of hyperglycaemia were $100 \%$, $33 \%, 40 \%$ and $0 \%$ for those receiving $125,75,50$ and 25 islets, respectively (Fig. 1b). Only recipients of 125 islets had fasting blood glucose levels significantly lower than shamtreated diabetic mice beginning 6 days post transplant; glucose levels in these mice were statistically indistinguishable from non-diabetic control mice between 12 and 47 days post transplant (Fig. 1c). Thus, transplant of 125 islets in the eye was sufficient to completely reverse fasting hyperglycaemia in insulin-dependent diabetic mice. In contrast, a dose of 125 islets transplanted under the kidney capsule of STZ-treated diabetic mice was insufficient to normalise blood glucose levels. A minimum of 200 islet equivalents was required to lower blood glucose when transplanted under the kidney capsule, but only 275 islets normalised blood glucose levels in a similar time frame to 125 islet equivalents in the eye (Fig. 1g).

Mice that received transplants of 50,75 or 125 islet equivalents gained weight faster than sham-treated mice $(p<0.05$, electronic supplementary material [ESM] Fig. 1 and Fig. 1d), indicating that transplantation of as few as 50 islets in the eye could restore weight gain in mice with STZ-induced diabetes. The growth rate in recipients of 125 islets was statistically indistinguishable from that of control mice ( $p>0.05$, ESM Fig. 1 and Fig. 1d). In contrast, only mice that received doses of 200 or 275 islets transplanted under the kidney capsule tended to gain weight (Fig. 1h).

An OGTT was performed to assess the ability of islet transplants to control glycaemia following a glucose challenge. Recipients of 75 or 125 islets in the eye showed nearnormal glucose tolerance, similar to that shown by nondiabetic control mice, except for slightly elevated peak postprandial glucose levels (Fig. 1e). At 47 days post transplantation, recipients of 125 islets that were subjected to an OGTT had their transplants removed via enucleation to determine whether the observed changes in glucose control were due to the direct action of the transplanted islets. Three non-diabetic control mice were also enucleated at the same time. Islet recipients rapidly became hyperglycaemic (blood glucose $\geq 25.4 \mathrm{mmol} / \mathrm{l}$ ) within 1 day of enucleation, indicating that these mice were highly dependent on the transplanted islets for glucose control (Fig. 1f).

The morphology of engrafted islets under the kidney capsule and in the anterior chamber of the eye was compared by immunofluorescent staining. In both transplant sites, the engrafted islets clustered and formed an endocrine cell mass containing insulin-producing beta cells and glucagonproducing alpha cells (Fig. 2). It is well established that islets transplanted under the kidney capsule become highly vascularised [27]. Islet grafts within the anterior chamber became highly vascularised within several days of transplantation, generally via a large vessel running through the transplanted area with many small branches supplying different graft regions (ESM Video 1).

Anterior chamber of the eye to evaluate autoimmune destruction of transplanted islets To compare autoimmune destruction of endogenous and engrafted allogeneic islets, NOD-SCID mice received donor NOD-SCID mouse islet transplants in the anterior chamber. To accelerate the natural process of autoimmune islet destruction, splenocytes from newly diabetic NOD mice were isolated for adoptive transfer into NOD-SCID islet transplant recipients. Engrafted islets were imaged in the eye four times before adoptive transfer of splenocytes and then regularly following the adoptive transfer to assess changes in islet vascularisation and morphology.

Morphological characteristics of the transplanted islets before and after adoptive transfer are described in Table 1, including the formation of ruffled edges (irregular, nonsmooth borders around transplanted pancreatic islets), holes in the transplant and changes in transparency. Islet transparency was graded on a scale of 0-3, ranging from no detectable transparency (grade 0 ) to greater than $50 \%$ transparency or evidence of holes (grade 3). Figure 3 and ESM Fig. 2 illustrate the progression of disease via representative images of transplants at different time points. Four weeks after the adoptive transfer, most of the islet grafts had decreased in size and developed areas of transparency and/or ruffled edges, possible indications of islet cell apoptosis (Fig. 3, Table 1). Moreover, subtle changes in islet morphology were visible as early as 19 days post adoptive transfer (Fig. 3, Table 1).

To determine whether autoimmune destruction of engrafted islets within the eye was also reflected in islets within the endogenous pancreas, we evaluated insulitis in the pancreas and engrafted eye sections from nine islet transplant recipient mice following adoptive transfer of splenocytes. We also examined engrafted eye and pancreas tissues from NOD mice undergoing spontaneous disease progression (i.e. no adoptive transfer), before the onset of diabetes. Interestingly, in both models, the pattern of insulitis was remarkably similar between endogenous islets in the pancreas and islets transplanted into the anterior chamber of the eye (Fig. 4). Insulitis scores for islets within the pancreas and the eye were significantly correlated ( $r=0.81, p<0.05$; Fig. 5), indicating that the degree of autoimmune attack on islets transplanted in the eye mirrors that in the pancreas. 
Fig. 2 Comparison of islets transplanted under the kidney capsule and in the anterior chamber of the eye. Immunofluorescent staining was carried out for insulin (red) and glucagon (green) in the kidney and eye sections of C57BL/6 mice transplanted with $\mathrm{C} 57 \mathrm{BL} / 6$ mouse islets. Nuclei were stained with DAPI (white). Scale bars: $1,000 \mu \mathrm{m}$ (kidney, low magnification), $500 \mu \mathrm{m}$ (eye, low magnification) and $50 \mu \mathrm{m}$ (lower panels)

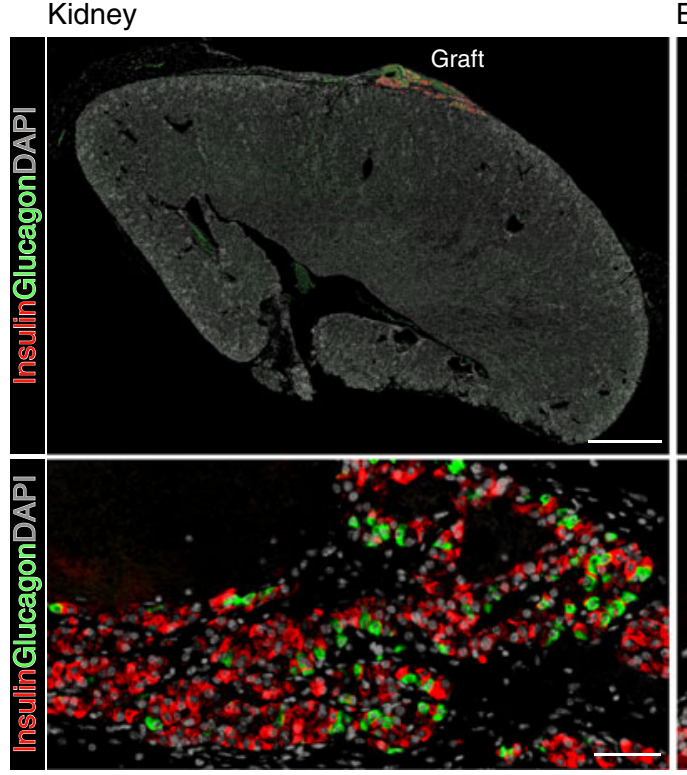

Eye

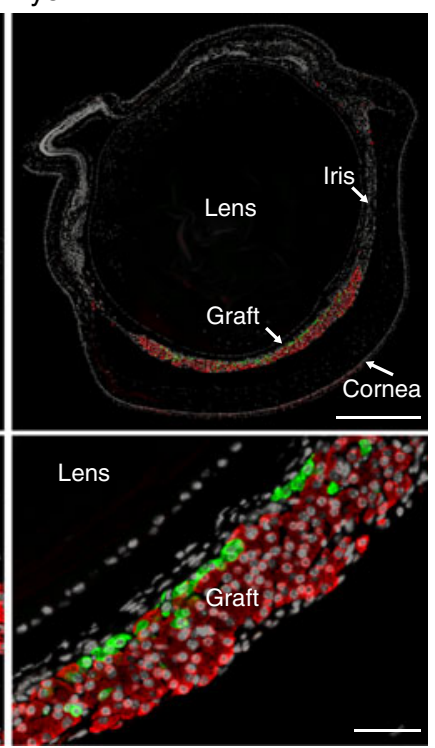

\section{Discussion}

In these studies we demonstrate that the anterior chamber of the eye is a highly effective site for functional islet transplantation, even more so than the kidney capsule transplant site traditionally used in animal models. Gross morphology and vascularisation of engrafted islets can easily be monitored in the eye with non-invasive imaging, as opposed to the kidney capsule, which requires a surgical procedure to externalise and visualise engrafted tissue. Our data demonstrate that autoimmune destruction of engrafted islets within the eye could be easily visualised and mirrored subclinical autoimmune processes occurring in endogenous pancreatic islets. These findings suggest a possible future avenue for mechanistic evaluation of islet autoimmunity using noninvasive in vivo imaging at cellular resolution.

Previous studies demonstrated that the anterior chamber of the eye was a safe and suitable site for islet engraftment in rodents [16, 17] and baboons [19]. The current study extends these findings to demonstrate that a remarkably low number of syngeneic islets transplanted in the eye yields significant physiological effects. Transplantation of as few as 50 islets in the eye improved survival of mice with diabetes, while transplantation of 125 islets completely reversed diabetic symptoms. More than double this number of islets was required to achieve the same effect when they were transplanted under the kidney capsule (Fig. 1g). The better outcome in the eye may be related to the rapid vascularisation that occurs at

Table 1 Characteristics of islets transplanted into the anterior chamber of the mouse eye before and over the course of diabetes following adoptive transfer of diabetic splenocytes

\begin{tabular}{|c|c|c|c|c|c|c|}
\hline \multirow[t]{2}{*}{ Mouse No. } & \multicolumn{2}{|c|}{ Adoptive transfer day } & \multicolumn{2}{|c|}{2 weeks post transfer } & \multicolumn{2}{|l|}{4 weeks post transfer } \\
\hline & Ruffling & Transparency & Ruffling & Transparency & Ruffling & Transparency \\
\hline 1 & None & 0 & None & 1 & Deceased at 2 weeks & Deceased at 2 weeks \\
\hline 2 & None & 0 & Yes & 0 & Yes & 3 \\
\hline 3 & None & 0 & None & 1 & None & 1 \\
\hline 4 & None & 0 & None & 2 & Yes & 3 \\
\hline 5 & None & 0 & Yes & 3 & Deceased at 2 weeks & Deceased at 2 weeks \\
\hline 6 & None & 0 & Yes & 0 & Yes & 2 \\
\hline 7 & None & 0 & Yes & 3 & Deceased at 2 weeks & Deceased at 2 weeks \\
\hline 8 & None & 0 & None & 3 & None & 3 \\
\hline 9 & None & 0 & None & 1 & None & 1 \\
\hline
\end{tabular}

Morphological characteristics of the transplanted islets before and after adoptive transfer are described, including the formation of ruffled edges (irregular, non-smooth borders around transplanted pancreatic islets), holes in the transplant and changes in transparency. Islet transparency was graded on a scale of $0-3$, ranging from no detectable transparency (grade 0 ) to greater than $50 \%$ transparency or evidence of holes (grade 3 ) 


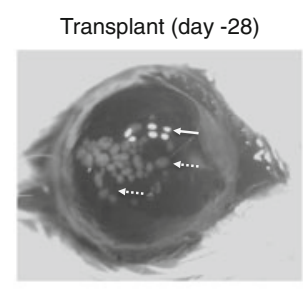

Day 19
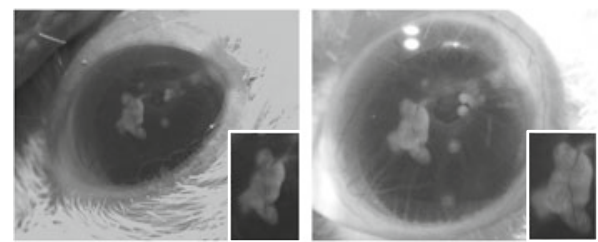

Fig. 3 Representative images of engrafted islets, pre and post adoptive transfer of splenocytes from diabetic NOD-SCID mice. Female NOD-SCID mice were transplanted with islets isolated from NOD-SCID mice into the anterior chamber of the eye. Four weeks (day 0 ) after the islet transplantation (performed on day -28), adoptive transfer of 20 million splenocytes from newly diabetic NOD mice was performed for

this site, which may promote better survival and engraftment of the transplanted cells compared with the kidney site. Current islet transplantation protocols in humans require islets to be isolated from two donor pancreases to treat a single patient, with recipient demand far exceeding donor supply [28]. The remarkable efficiency we observed when transplanting islets into the eye of rodents suggests that additional optimisation of

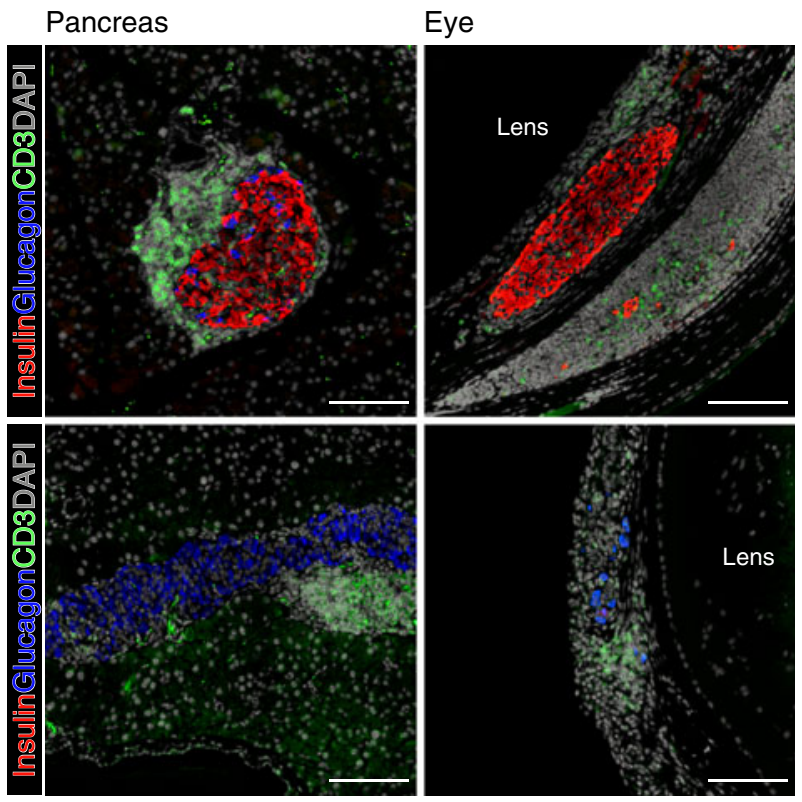

Fig. 4 Different stages of insulitis in the pancreas and in the eye in two mice. Immunofluorescent staining for insulin (red), glucagon (blue) and CD3 (green) is shown in the pancreas and eye of an NOD-SCID mouse post adoptive transfer of splenocytes. Merged images are shown of islets with an insulitis score of 2 (peri-insulitis, upper panels) and 3 (severe insulitis, lower panels). Nuclei are stained with DAPI (white). Scale bars: $100 \mu \mathrm{m}$

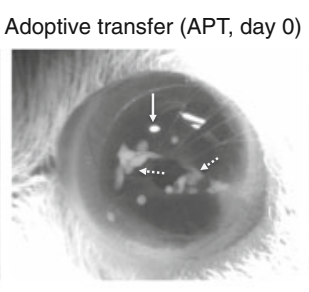

Day 25

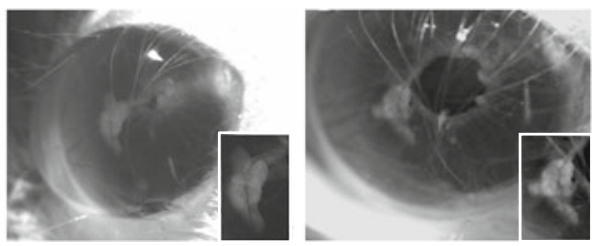

each islet transplant recipient. Images of engrafted islets were taken at three time points before adoptive transfer (day -28 , day -8 and day 0 ) and at five time points after adoptive transfer (days 5, 19, 21, 25 and 28). Note: in some images overhead lights are reflected in the cornea (indicated by solid arrows). Representative islets or islet clusters are marked with dotted arrows. Inset images are $\times 1.5$ magnification of selected area

current islet transplantation protocols may enable a drastic decrease in the islet volumes required for successful treatment of diabetes in humans.

A clear advantage of the eye over traditional transplant sites is the ability to non-invasively monitor the transplanted cells over time. We used in vivo imaging to demonstrate that engrafted islets remain intact in distinct clusters following transplantation, and that the graft tissue was vascularised within a few days of transplantation. This agrees with previous observations that transplanted tissue in the anterior chamber of the eye becomes vascularised within $24 \mathrm{~h}$ [29]. We predicted that the destruction of islets transplanted into the eye might occur in tandem with autoimmune destruction of pancreatic islets. To test this we monitored natural diabetes development and diabetes development following

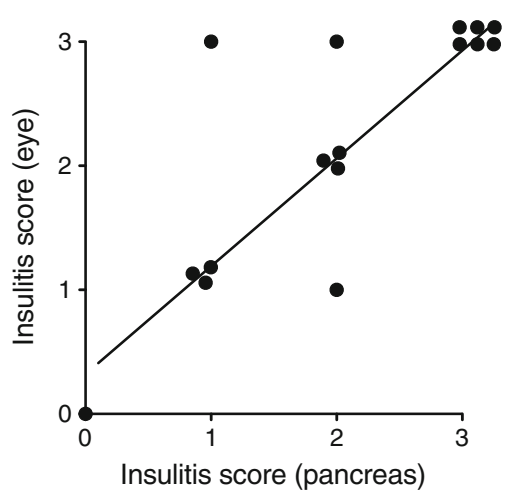

Fig. 5 Insulitis scoring of the transplanted tissue in the eye as compared with the pancreas. Scoring of insulitis in the eye vs the pancreas from individual NOD and NOD-SCID mice was evaluated in samples from two studies $(n=16)$. Seven of 16 mice had diabetes at the time of tissue harvest. Insulitis scores for islets within the pancreas and the eye were significantly correlated $(r=0.81, p<0.05)$ 
adoptive transfer of diabetogenic splenocytes in NOD-SCID mice. We chose not to use reporter proteins so that we could exclude any possibility of immune responses toward nonself proteins. Our data support the notion that autoimmune destruction of islets occurs simultaneously in the eye and in the pancreas in this model.

The strong correlation between the degree of islet destruction in the pancreas and eye support our hypothesis that in vivo imaging of islet grafts in the eye can enable realtime tracking of diabetes progression in the pancreas. As early as 2 weeks following transfer of splenocytes from newly diabetic NOD mice, we observed subtle changes in islet morphology that were indicative of islet destruction. When the mice were killed, we confirmed that immune cell infiltration was present in islets in both the pancreas and the eye, suggesting that disease progression was underway even though these mice were not yet overtly hyperglycaemic.

Abdulreda et al [18] showed the feasibility of studying immune attack of transplanted islets in the anterior chamber of the eye. They transplanted pancreatic islets with mismatched major histocompatibility complex into the eye. By tracking immune cell infiltration into the engrafted tissue via fluorescent $\mathrm{T}$ cells, they demonstrated the practicality of this model for visualising islet allograft rejection. However, they did not address whether the eye is an appropriate site to evaluate islet autoimmunity. Our study shows proof of principle that anterior chamber islet transplantation can enable visualisation and monitoring of autoimmune attack against pancreatic islets and suggests that this model may be appropriate for studying interventions that could modify disease progression. In our study we did not incorporate any fluorescent reporter in either islets or T cells to directly evaluate autoimmune attack of pancreatic islets and rule out the possibility of immune attack against reporter proteins. Instead, we illustrated with basic, non-fluorescent imaging the feasibility of using the eye as a model to visualise diabetic autoimmune attack. Future studies could use fluorescent reporters to track autoimmune processes in greater detail.

Immune privilege within the eye is thought to be an evolutionary adaptation designed to protect the eye from bystander effects of inflammation. However, under some circumstances ocular immune privilege is lost (e.g. allogeneic cornea grafts are no longer protected from immune rejection [30]). The current study and that of Abdulreda et al [18] support the notion that pancreatic islets are not protected from autoimmune processes when transplanted into the anterior chamber. This breaching of the normal immune privileged status of the anterior chamber may be due to mechanical damage during surgical procedures and/or due to angiogenic factors from transplanted islets that lead to revascularisation of the islet graft, making it accessible to the recipient's immune system.

Our studies provide compelling evidence supporting the eye as an excellent site for functional islet transplantation to treat diabetes and also to monitor survival of transplanted islets in real time. Importantly, we demonstrated that the process of autoimmune destruction of islets in the eye reflects that occurring within the endogenous pancreas, a site that cannot be visualised non-invasively using current imaging modalities. To our knowledge, this is the first study to demonstrate a means to track progression of beta cell autoimmunity over the course of type 1 diabetes development. The eye provides a unique opportunity for future studies using fluorescent probes in vivo to identify the phenotypes of the cells attacking the islets in real time as well as to investigate novel therapeutic strategies.

Acknowledgements The authors acknowledge editorial support from Rhonda Wideman, Life Sciences Institute, University of British Columbia, Vancouver, BC, Canada.

Funding Majid Mojibian and Jennifer E. Bruin are grateful recipients of JDRF postdoctoral fellowships and awards from the Canadian Institutes of Health Research/Michael Smith Foundation for Health Research (CIHR/MSFHR) Strategic Training Program in Transplant Research. Jennifer E. Bruin is a recipient of the L'Oreal Canada For Women in Science Research Excellence Fellowship. Timothy J. Kieffer was a Senior Scholar of the MSFHR. This project received funding support from the JDRF and the Canadian Diabetes Association.

Duality of interest The authors declare that there is no duality of interest associated with this manuscript.

Contribution statement $\mathrm{MM}, \mathrm{BH}, \mathrm{AH}, \mathrm{AA}$ and JEB designed the experimental approach and acquired, analysed and interpreted data. $\mathrm{MM}$ and $\mathrm{BH}$ drafted the manuscript. TJK designed the experimental approach and contributed to discussion and revising the manuscript critically for intellectual content. All authors revised and approved the final manuscript.

\section{References}

1. Waldron-Lynch F, Herold KC (2011) Immunomodulatory therapy to preserve pancreatic beta-cell function in type 1 diabetes. Nat Rev Drug Discov 10:439-452

2. Ryan EA, Paty BW, Senior PA et al (2005) Five-year follow-up after clinical islet transplantation. Diabetes 54:2060-2069

3. Robertson RP (2004) Islet transplantation as a treatment for diabetes - a work in progress. N Engl J Med 350:694-705

4. Dy EC, Harlan DM, Rother KI (2006) Assessment of islet function following islet and pancreas transplantation. Curr Diab Rep 6:316322

5. Ris F, Lepetit-Coiffe M, Meda P et al (2010) Assessment of human islet labeling with clinical grade iron nanoparticles prior to transplantation for graft monitoring by MRI. Cell Transplant 19:15731585

6. Fan Z, Spencer JA, Lu Y et al (2010) In vivo tracking of "colorcoded' effector, natural and induced regulatory T cells in the allograft response. Nat Med 16:718-722

7. Toso C, Zaidi H, Morel P et al (2005) Positron-emission tomography imaging of early events after transplantation of islets of Langerhans. Transplantation 79:353-355 
8. Medarova Z, Vallabhajosyula P, Tena A et al (2009) In vivo imaging of autologous islet grafts in the liver and under the kidney capsule in non-human primates. Transplantation 87:1659-1666

9. Evgenov NV, Medarova Z, Dai G, Bonner-Weir S, Moore A (2006) In vivo imaging of islet transplantation. Nat Med 12:144-148

10. Virostko J, Radhika A, Poffenberger G et al (2009) Bioluminescence imaging in mouse models quantifies beta cell mass in the pancreas and after islet transplantation. Mol Imaging Biol 12:42-53

11. Virostko J, Radhika A, Poffenberger G, Dula AN, Moore DJ, Powers AC (2013) Bioluminescence imaging reveals dynamics of beta cell loss in the non-obese diabetic (NOD) mouse model. PLoS One 8:e57784

12. Virostko J, Henske J, Vinet L et al (2011) Multimodal image coregistration and inducible selective cell ablation to evaluate imaging ligands. Proc Natl Acad Sci U S A 108:20719-20724

13. Altinoglu EI, Adair JH (2010) Near infrared imaging with nanoparticles. Wiley Interdiscip Rev Nanomed Nanobiotechnol 2:461-477

14. Ntziachristos V (2010) Going deeper than microscopy: the optical imaging frontier in biology. Nat Methods 7:603-614

15. Moore RA, Rosenblum HB, Tolins SH, Melchionna RH (1937) Variation in the size of transplants of the prostate and seminal vesicle in the anterior chamber of the eye. J Exp Med 66:273-280

16. Speier S, Nyqvist D, Kohler M, Caicedo A, Leibiger IB, Berggren PO (2008) Noninvasive high-resolution in vivo imaging of cell biology in the anterior chamber of the mouse eye. Nat Protoc 3:1278-1286

17. Speier S, Nyquist D, Cabrera O et al (2008) Noninvasive in vivo imaging of pancreatic islet cell biology. Nat Med 14:574-578

18. Abdulreda MH, Faleo G, Molano RD et al (2011) High-resolution, noninvasive longitudinal live imaging of immune responses. Proc Natl Acad Sci U S A 108:12863-12868
19. Perez VL, Caicedo A, Berman DM et al (2011) The anterior chamber of the eye as a clinical transplantation site for the treatment of diabetes: a study in a baboon model of diabetes. Diabetologia 54:1121-1126

20. Streilein JW (2003) Ocular immune privilege: therapeutic opportunities from an experiment of nature. Nat Rev Immunol 3:879-889

21. Ferguson TA, Herndon JM (1994) The immune response and the eye: the ACAID inducing signal is dependent on the nature of the antigen. Invest Ophthalmol Vis Sci 35:3085-3093

22. Taylor AW (2009) Ocular immune privilege. Eye (Lond) 23:1885-1889

23. Coppieters K, Amirian N, von Herrath M (2012) Intravital imaging of CTLs killing islet cells in diabetic mice. J Clin Invest 122:119-131

24. Lacy PE, Kostianovsky M (1967) Method for the isolation of intact islets of Langerhans from the rat pancreas. Diabetes 16:35-39

25. Salvalaggio PR, Deng S, Ariyan CE et al (2002) Islet filtration: a simple and rapid new purification procedure that avoids ficoll and improves islet mass and function. Transplantation 74:877-879

26. Riedel MJ, Asadi A, Wang R, Ao Z, Warnock GL, Kieffer TJ (2012) Immunohistochemical characterisation of cells co-producing insulin and glucagon in the developing human pancreas. Diabetologia 55:372-381

27. Morini S, Brown ML, Cicalese L et al (2007) Revascularization and remodelling of pancreatic islets grafted under the kidney capsule. J Anat 210:565-577

28. Cravedi P, van der Meer IM, Cattaneo S, Ruggenenti P, Remuzzi G (2010) Successes and disappointments with clinical islet transplantation. Adv Exp Med Biol 654:749-769

29. Adeghate E (1998) Host-graft circulation and vascular morphology in pancreatic tissue transplants in rats. Anat Rec 251:448-459

30. Streilein JW (2003) Ocular immune privilege: the eye takes a dim but practical view of immunity and inflammation. J Leukoc Biol 74:179-185 